\title{
Uncooled infrared detector designed for Gas detection and High temperature measurements
}

\author{
Arnaud Crastes, Aurélie Touvignon, Sophie Bethoux-Garidel, Sébastien Tinnes \\ ULIS - ZI Les Cordées - 38113 VEUREY-VOROIZE - FRANCE \\ a.crastes@ulis-ir.com \\ ULIS - ZI Les Cordées - 38113 VEUREY-VOROIZE - FRANCE
}

\begin{abstract}
:
Uncooled infrared focal plane arrays are being developed for a wide range of thermal imaging applications. A new type of detector for middle and long wave applications was developed based on amorphous silicon VGA device. This new detector is well adapted for gas detection, high temperature measurements, and special industrial processes (boiler control and maintenance). After a brief introduction to microbolometer technological background, the interest of such detectors will be presented by comparing LWIR / MWIR detector signal to noise ratio, and the advantages for different applications will be highlighted. Finally, the main electro-optical characteristics and performances will be presented.
\end{abstract}

Key words: uncooled, microbolometer, MWIR, spectral measurement, absorption

\section{Introduction}

For the last two decades, uncooled infrared detection has run the race towards pixel pitch reduction down to $12 \mu \mathrm{m}$ at R\&D level for the time being and expected to be in production soon. A new era is now starting with additional features or innovation at the Read-Out Integrated Circuit (ROIC) level, or addressing multispectral imaging: LWIR and visible or LWIR and MWIR on the same chip.

Uncooled detectors have significant potential capabilities that have so far not been extensively explored [1]. Cooled detectors completely dominate narrowband sensing applications, such as chemical identification. This is probably because thermal detectors have traditionally been designed to absorb across the entire thermal infrared spectrum and the materials traditionally chosen to absorb do not lend themselves to spectral selectivity. In this paper, micromachined uncooled detectors with spectral characteristics from MWIR to LWIR (3 to $16 \mu \mathrm{m})$ are described. We further describe related results on the optical quarter wave optical cavity characteristics of microbolometers. We conclude with some sensitivity results in terms of NEP for different spectral narrowband and we give some examples of targeted applications.

\section{Optical quarter wave cavity engineering}

Amorphous silicon can be easily integrated onto silicon substrates at temperatures compatible with CMOS integrated circuit. Advanced lithography in conjunction with thinner films embodiment clearly give a way to maintain, and even to improve, the thermal insulation. Scaling the pixel to $17 \mu \mathrm{m}$ while keeping a simple one level microbridge structure leads to high operability and high manufacturing yield. This can be achieved without any need for complex and space damaging wrap around and/or meandering leg structures. As a result, legs space arrangement remains quite undemanding and a high fill factor can be maintained. This approach allows to continue to scale down a single level microbolometer architecture, avoiding unwanted spectral artifacts caused by coupling of different cavity heights which could interfere with each other in case of umbrella architecture. The figure 2 below gives the theoretical spectral absorption obtained with such simple one deck design.

This simulation is based on:

- infinite pixel size: no perfectly matched layer boundary conditions required,

- planar layers: both ROIC and pixel structure are flat as shown on the SEM picture below. 


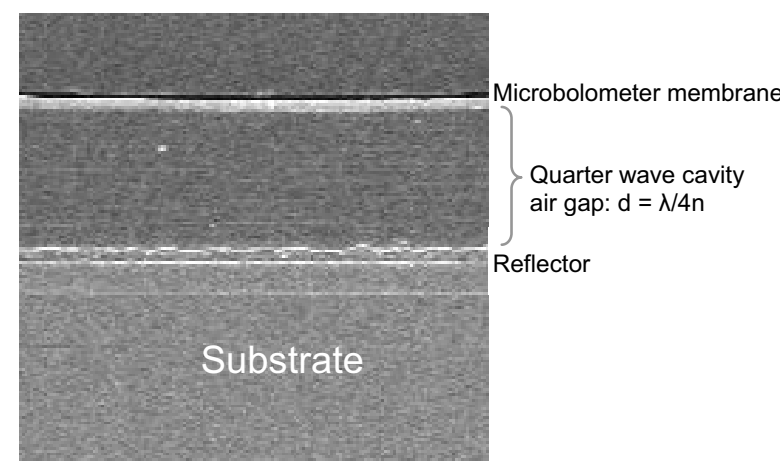

Fig. 1. SEM picture magnification showing the air optical quarter wave cavity and the layers' flatness

- material characteristics: the different layers, reflector, various material forming the stack, are simulated through their refraction index and absorption coefficient [2].

- plane wave: the incident wave is regarded as a plane wave coming from the infinite. In real measurement, many plane waves arriving with different incident angles must be considered.

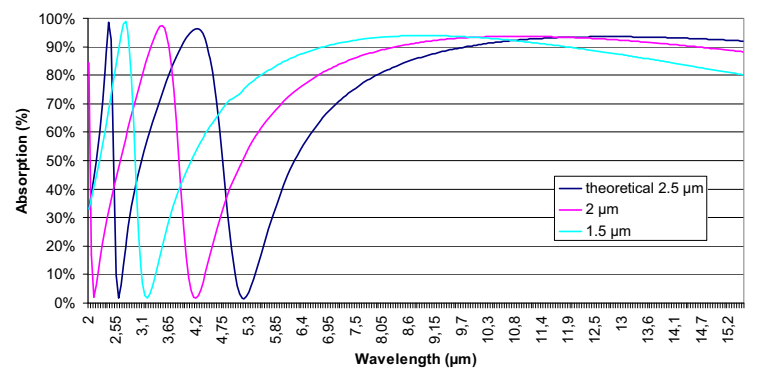

Fig. 2. Theoretical spectral response of a quarter optical cavity with different gap thicknesses

According to the wavelength of interest, the first minimum absorption curve value is given by: $\alpha(\lambda=2 d)=\alpha_{\min } \sim 0$, where $\alpha$ is the absorption coefficient and $d$ the cavity height. This simple rule can be a help for early stage design.

The ROIC top layer and reflector geometry can be one way to adjust spectral sensitivity. Indeed the planarization of the ROIC is a degree of freedom which can be used to modulate the global wavelength sensitivity. The figure 3 below gives the first generation spectral sensitivity using a non-planar ROIC topology.

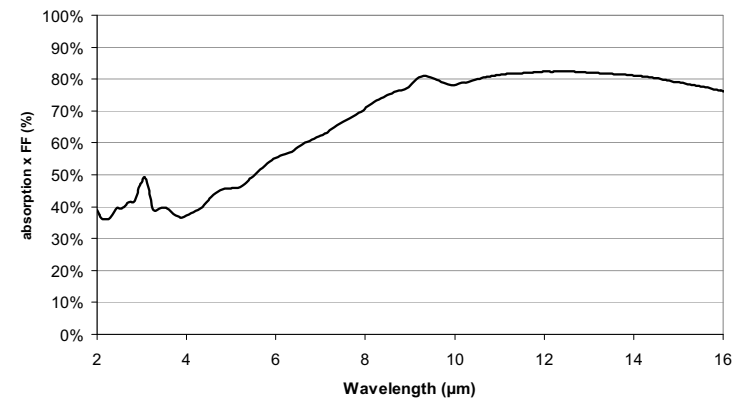

Fig. 3. Spectral response of the previous generation $(35 \mu \mathrm{m})$ broadband microbolometer

The advantage of a non-planar topology is to obtain an overall average sensitivity, mainly in the MWIR, without any loss of absorption whatever the wavelength, but without either any large sensitivity in the MWIR spectral range. The trade-off between surface structure and MWIR spectral response has to be considered finely according to targeted applications. For this new device, the present approach was to design a cavity having a maximum response around $3.9 \mu \mathrm{m}$. Consequently, the design with a planar reflector was preferred.

\section{Spectral measurement and results}

Taking into account theoretical model, different cavities were designed enabling to detect infrared radiations from 3 to $16 \mu \mathrm{m}$. The following figure gives FTIR measurement results.

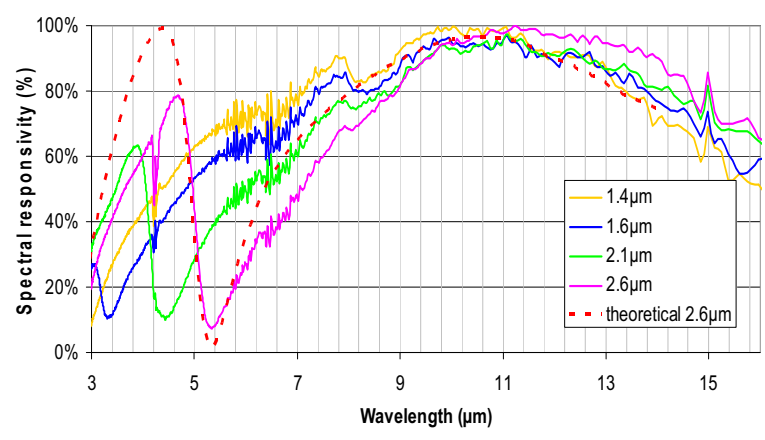

Fig. 4. Spectral response of the $640 \times 480 / 17 \mu m$ broadband detector according to cavity gap

The cavity height mainly impacts the spectral response in the lower wavelengths region (MWIR) and does not vary too much in the LWIR. Each cavity height shows an interest according to the targeted spectral range. For instance, the highest cavity height is more interesting for MWIR detection while the short cavity will be dedicated to detection around 7 to 8 micrometers.

On figure 4, the comparison between theoretical simulation taking into account window transmission (dotted curve), and FTIR measurement for a cavity height of $2.6 \mu \mathrm{m}$ is also given. According to previous 
considerations, the matching is rather good and the maximum and minimum curve fit quite well.

\section{High temperature measurement}

A complete electro-optical characterization has been carried out at different blackbody temperatures giving the transition temperature where the signal to noise ratio is higher in MWIR compared to the LWIR. Then, we are looking for the temperature (T) leading to:

$$
\int_{3}^{5} L(\lambda, T) R(\lambda) \tau(\lambda) d \lambda>\int_{8}^{12} L(\lambda, T) R(\lambda) \tau(\lambda) d \lambda
$$

where $L$ is the Planck' law, $R$ is the detector sensitivity and $\tau$ the window transmission. The next four graphs give, for each cavity height, the relative value of the previous quantity in both spectral bands. From $400^{\circ} \mathrm{C}$ scene temperature, the MWIR selection will lead to higher signal to noise ratio.

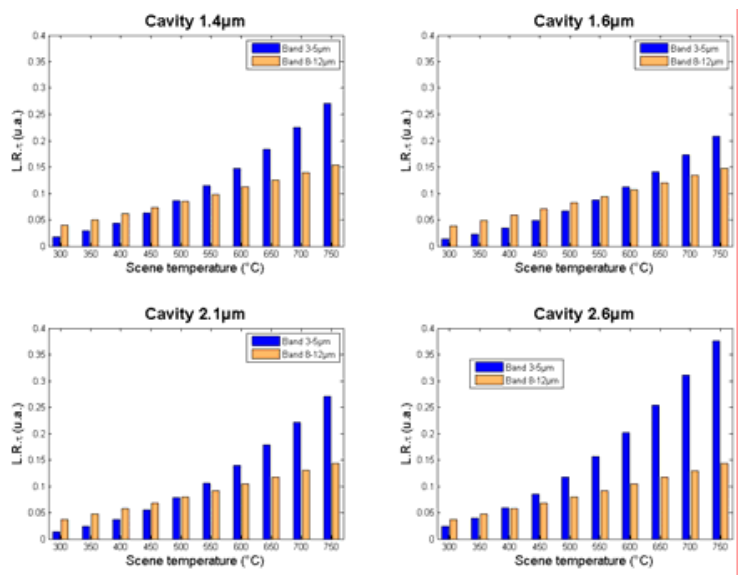

Fig. 3. Signal to noise ratio for different scene temperatures, spectral band and cavity height

\section{"Through flame" application}

Moreover, some measurements have been carried out at $3.9 \mu \mathrm{m}$, addressing "through flame" applications, and leading to a Noise Equivalent Power (NEP) around 50pW. To do this test, a filter, with a $110 \mathrm{~nm}$ width (FWHM) and a peak value of $80 \%$, is added in the optical path. Using two DC level acquisitions in front of $20^{\circ} \mathrm{C}$ and $500^{\circ} \mathrm{C}$, the responsivity and NEP were determined using the following equations:

$$
\begin{aligned}
& R=\frac{\Delta \text { DClevel }_{500^{\circ} \mathrm{C}-20^{\circ} \mathrm{C}}}{\Delta L_{500^{\circ} \mathrm{C}-20^{\circ} \mathrm{C} \_ \text {filter } 3.9 \mu \mathrm{m}}} \cdot \frac{1}{S_{\text {pixel }}} \cdot \frac{\pi}{4 N^{2}} \\
& N E P=\frac{\text { temporal_noise }}{R}
\end{aligned}
$$

Where $S_{\text {pixel }}$ is the pixel area $\left(17 \times 17 \mu \mathrm{m}^{2}\right)$ and $\mathrm{N}$ the optical F-number $(\mathrm{N}=1)$ used during the experiment.
Tab. 1: Impact of cavity height on NEP

\begin{tabular}{|c|c|c|}
\hline $\begin{array}{c}\text { Cavity } \\
\text { height } \\
(\mu \mathrm{m})\end{array}$ & $\begin{array}{c}\text { Resp (V/W) / } \\
\text { absorption @ } \\
3.9 \mu \mathrm{m}\end{array}$ & $\begin{array}{c}\text { NEP }(\mathrm{pW}) \\
@ 3.9 \mu \mathrm{m}\end{array}$ \\
\hline 1.4 & $1.8010^{7} / 40 \%$ & 74 \\
\hline 1.6 & $1.5310^{7} / 30 \%$ & 90 \\
\hline 2.1 & $2.5010^{7} / 62 \%$ & 56 \\
\hline 2.6 & $2.6410^{7} / 58 \%$ & 51 \\
\hline
\end{tabular}

These NEP values are directly correlated to the relative absorption at 3.9 micrometers.

In addition, we can compare the NEP value obtained with $17 \mu \mathrm{m}$ pixel pitch with ULIS former generation of broadband detector $(35 \mu \mathrm{m})$. The same experiment as previously described is used, led to a NEP @3.9 $\mu \mathrm{m}$ of $820 \mathrm{pW}$ for $35 \mu \mathrm{m}$ pixel pitch. Thus the new broadband detector based on $17 \mu \mathrm{m}$ technology is 10 times more sensitive than the previous $35 \mu \mathrm{m}$ for the same cavity height. This result is due to continuous performance improvement of the technology itself while pixel is shrinking. Indeed for $17 \mu \mathrm{m}$ technology, the NETD in 8 to $14 \mu \mathrm{m}$ spectral range is typically $40 \mathrm{mK}$ with a thermal time constant lower than $10 \mathrm{~ms}$ allowing an operation up to $50 \mathrm{~Hz}$ frame rate. As mentioned in the first part, this can be possible thanks to one deck design as well as very thin pixel layer due to the use of amorphous silicon (see picture in figure 1).

\section{Leakage gas application}

Some applications like gas leakage detection are more demanding in terms of sensitivity, while the spatial resolution or frame rate are not primary figures of merit. Accordingly measurements using frame averaging and pixel binning $(2 \times 2)$ have been carried out leading to an improvement of the signal to noise ratio in the range of $4 \mathrm{x}$. The table below shows the improvement on the temporal noise after a $2 \times 2$ binning and the importance of the detector settings. Indeed, with basic setting, only a factor of $1.8 \mathrm{x}$ instead of 2 can be achieved due to limitation by the floor ROIC, 1/f and system acquisition noise which is in the range of $200 \mu$ Vrms.

Tab. 2: Temporal noise evolution with binning

\begin{tabular}{|c|c|c|}
\hline $\begin{array}{c}\text { Test } \\
\text { conditions }\end{array}$ & $\begin{array}{c}\text { Temporal } \\
\text { noise }(\mu \mathrm{Vrms})\end{array}$ & $\begin{array}{c}\text { Temporal noise }(\mu \mathrm{Vrms}) \\
\text { after } 2 \times 2 \text { binning }\end{array}$ \\
\hline $\begin{array}{c}\text { Nominal } \\
\text { setting }\end{array}$ & 417 & 223 \\
\hline
\end{tabular}

The binning application on VGA format with a pixel pitch of $17 \mu \mathrm{m}$ leads to a QVGA with an equivalent pitch of $34 \mu \mathrm{m}$ close to the $35 \mu \mathrm{m}$ of the previous generation. Consequently, an 
optical lens previously developed can be reused keeping the same field of view.

Current experiments are carried out to detect gas leaks of hydrocarbons (methane, propane or butane) or other gases like $\mathrm{SF}_{6}$ or $\mathrm{NH}_{4}$.

\section{Conclusions and future developments}

This paper gives an overview of the choice targeted during the design phase. Spectral characterization has been carried out fitting well with design phase expectations. The performance improvement, more than 10 times better compared to previous broadband generation will open new markets opportunities mainly in gas leak detection. Future long term developments would consider pixel wavelength selectivity at pixel level, leading to high flat absorption all along the array from MWIR to LWIR. This could be done, for example, using the additional cap layers used to make the vacuum at the pixel level: pixel level packaging techniques [3].

\section{References}

[1] B. Fieque, A. Crastes and al. "MWIR uncooled microbolometer: a way to increase the number of applications," Proc. SPIE 5783, 531-538 (2005).

[2] Kevin Liddiard, "application of interferometeric enhancement to self absorbing thin film thermal IR detectors", IR physics Vol 34 n4 p379-387 (1993).

[3] Dumont G. et al, "Current progress on pixel level packaging for uncooled IRFPA", Infrared technology and Applications XXXVIII, Proc. SPIE Vol. 8353, (2012). 